\title{
ON THE $n$-FOLD PSEUDO-HYPERSPACE SUSPENSIONS OF CONTINUA
}

\author{
JuAN CARLOS MACÍAS
}

Benemérita Universidad Autónoma de Puebla, Mexico

\begin{abstract}
Let $X$ be a (metric) continuum. Let $n$ be a positive integer, let $C_{n}(X)$ denote the space of all nonempty closed subsets of $X$ with at most $n$ components and let $F_{1}(X)$ denote the space of singletons. The $n$-fold pseudo-hyperspace suspension of $X$ is the quotient space $C_{n}(X) / F_{1}(X)$. We present properties of this hyperspace.
\end{abstract}

\section{INTRODUCTION}

In 1979 Sam B. Nadler Jr., introduced the hyperspace suspension of a continuum, $C(X) / F_{1}(X)[18]$. In 2004 Sergio Macías, gave a natural generalization of such hyperspace and he defined the $n$-fold hyperspace suspension of a continuum $X$ as the quotient space $C_{n}(X) / F_{n}(X)[12]$.

Now, we introduce the $n$-fold pseudo-hyperspace suspension of a continuum $X$ as the quotient space $C_{n}(X) / F_{1}(X)$ topologized with the quotient topology. The purpose of this paper is to present a study of these hyperspaces. The paper is divided into six sections. In section 2, we give the basic definitions necessary for understanding the paper. In section 3, we present some general properties of the $n$-fold pseudo-hyperspace suspensions of continua. In section 4 , we present results about the $n$-fold pseudo-hyperspace suspensions of locally connected continua. In section 5 , we show that the $n$-fold pseudo-hyperspace suspensions of continua are zero-dimensional aposyndetic. In section 6 , we show that a continuum $X$ is indecomposable if and only if by removing two specific points from the $n$-fold pseudo-hyperspace suspension of $X$ it becomes arcwise disconnected.

2000 Mathematics Subject Classification. 54B20.

Key words and phrases. Absolute retract, aposyndesis, Cantor manifold, continuum, hyperspace, indecomposable continuum, $n$-fold hyperspace, $n$-fold hyperspace suspension, $n$-fold pseudo-hyperspace suspension, unicoherent continuum. 


\section{Definitions}

If $(Y, d)$ is a metric space, then given $A \subset Y$ and $\varepsilon>0$, the open ball about $A$ of radious $\varepsilon$ is denoted by $\mathcal{V}_{\varepsilon}^{d}(A)$; that is:

$$
\mathcal{V}_{\varepsilon}^{d}(A)=\{y \in Y: d(y, a)<\varepsilon \text { for some } a \in A\} .
$$

We write $\mathcal{V}_{\varepsilon}(A)$ when the metric is understood.

The interior of $A$ is denoted by $\operatorname{Int}_{Y}(A)$, and the closure of $A$ is denoted by $C l_{Y}(A)$. We omit the subindices when there is no confusion. A map means a continuous function.

A continuum is a nonempty compact, connected metric space. A subcontinuum of a space $Y$ is a continuum contained in $Y$. A continuum is said to be decomposable provided that it can be written as the union of two of its proper subcontinua. A continuum is indecomposable if it is not decomposable. A continuum is said to have property $(b)$ provided that any map from $X$ into the unit circle $S^{1}$ is homotopic to a constant map. A continuum $X$ is unicoherent provided that if $X=A \cup B$, where $A$ and $B$ are subcontinua of $X$, then $A \cap B$ is connected. A continuum $X$ is uniformly pathwise connected provided that it is the continuous image of the cone over the Cantor set $[7$, 3.5].

An arc is any space homeomorphic to $[0,1]$. The end points of an $\operatorname{arc} \alpha$ are the image of 0 and 1 under any homeomorphism from $[0,1]$ onto $\alpha$. An $n$-cell is any space homeomorphic to $[0,1]^{n}$.

Given a continuum $X$ and a positive integer $n, C_{n}(X)$ denotes the $n$-fold hyperspace of $X$; that is:

$C_{n}(X)=\{A \subset X: A$ is nonempty, closed and has at most $n$ components $\}$, topologized with the Hausdorff metric defined as follows:

$$
\mathcal{H}(A, B)=\inf \left\{\varepsilon>0: A \subset \mathcal{V}_{\varepsilon}(B) \text { and } B \subset \mathcal{V}_{\varepsilon}(A)\right\},
$$

$\mathcal{H}$ always denotes the Hausdorff metric.

The symbol $F_{n}(X)$ denotes the $n$-fold symmetric product of $X$; that is:

$$
F_{n}(X)=\{A \subset X: A \text { has at most } n \text { points }\} .
$$

We agree that $C(X)=C_{1}(X)$.

It is known that $C_{n}(X)$ is an arcwise continuum (for $n=1$ see [17, (1.12)], for $n \geq 2$ see $[10,(3.1)])$.

Notation 2.1. Let $U_{1}, \ldots, U_{m}$ be nonempty subsets of a continuum $X$, we define

$$
\begin{aligned}
& \left\langle U_{1}, \ldots, U_{m}\right\rangle= \\
& \left\{A \in C_{n}(X): A \subset \bigcup_{k=1}^{m} U_{k} \text { and } A \cap U_{k} \neq \emptyset \text { for each } k \in\{1, \ldots, m\}\right\} .
\end{aligned}
$$


It is known that $C_{n}(X)$ can be topologized with the Vietoris Topology (see $[17,(0.11)])$, a base of which is given by:

$\mathcal{B}=\left\{\left\langle U_{1}, \ldots, U_{m}\right\rangle: U_{1}, \ldots, U_{m}\right.$ are nonempty open subsets of $\left.X\right\}$.

Also, it is known that the Vietoris Topology and the topology induced by the Hausdorff metric coincide [17, (0.13)].

An order arc in $C_{n}(X)$ is a one-to-one map $\alpha:[0,1] \rightarrow C_{n}(X)$ such that if $0 \leq s<t \leq 1$ then $\alpha(s) \subset \alpha(t)$ and $\alpha(s) \neq \alpha(t)$. If we have an order arc $\alpha$, we use the notation $\alpha$ to refer to the map $\alpha$ or to the image of the map $(\alpha([0,1]))$, when there is no confusion.

By the $n$-fold hyperspace suspension of a continuum $X$, denoted by $H S_{n}(X)$, we mean the quotient space:

$$
H S_{n}(X)=C_{n}(X) / F_{n}(X),
$$

with the quotient topology. Remark that $H S_{1}(X)$ corresponds to the hyperspace suspension $H S(X)$ defined by Nadler in [18]. Thus, for the rest of the paper we assume that $n$ is an integer such that $n \geq 2$.

By the $n$-fold pseudo-hyperspace suspension of a continuum $X$, denoted by $\operatorname{PH}_{n}(X)$, we mean the quotient space:

$$
P H S_{n}(X)=C_{n}(X) / F_{1}(X),
$$

with the quotient topology. The fact that $P H S_{n}(X)$ and $H S_{n}(X)$ are continua follows from $[19,3.10]$.

Notation 2.2. Given a continuum $X, q_{X}^{n}: C_{n}(X) \rightarrow P H S_{n}(X)$ denotes the quotient map. Also, $q_{X}^{n}\left(F_{1}(X)\right)=\left\{F_{X}^{n}\right\}$ and $q_{X}^{n}(X)=T_{X}^{n}$.

Remark 2.3. Note that $P H S_{n}(X) \backslash\left\{F_{X}^{n}\right\}$ and $P H S_{n}(X) \backslash\left\{T_{X}^{n}, F_{X}^{n}\right\}$ are homeomorphic to $C_{n}(X) \backslash F_{1}(X)$ and $C_{n}(X) \backslash\left(\{X\} \cup F_{1}(X)\right)$, respectively.

A free arc in a continuum $X$ is an $\operatorname{arc} \beta$ in $X$ such that $\beta \backslash$ end points\} is open in $X$.

A Hilbert cube is any space homeomorphic to $\mathcal{Q}=\prod_{n=1}^{\infty}[0,1]_{n}$ with the product topology, where $[0,1]_{n}=[0,1]$ for each positive integer $n$.

Other definitions are given as required.

\section{General properties}

Let $Y$ be a metric, compact space with the metric $d$. A closed subset $A$ of $Y$ is said to be a $Z$-set in $Y$ provided that for each $\varepsilon>0$, there is a continuous function $f_{\varepsilon}$ from $Y$ into $Y \backslash A$ such that $f_{\varepsilon}$ is within $\varepsilon$ of the identity map on $Y$ (i.e., $d\left(f_{\varepsilon}(y), y\right)<\varepsilon$ for all $\left.y \in Y\right)[5$, p. 78$]$.

Lemma 3.1. $F_{1}([0,1])$ is a $Z$-set in $C_{2}([0,1])$. 
Proof. Let $\varepsilon>0, I=[0,1]$ and let $f_{\varepsilon}: C_{2}(I) \rightarrow C_{2}(I) \backslash F_{1}(I)$ given by $f_{\varepsilon}(A)=C l\left(\mathcal{V}_{\frac{\varepsilon}{2}}^{d}(A)\right)$, it is easy to see that $f_{\varepsilon}$ satisfies the requires properties.

Note that by [4, Lemma 2.2], $C_{2}(I)$ is a 4-cell. Hence, we have the following:

COROllary 3.2. $F_{1}([0,1])$ is a subset of the manifold boundary of $C_{2}([0,1])$.

Proof. Let $\beta$ be the manifold boundary of $C_{2}([0,1])$. Since $F_{1}([0,1])$ is a Z-set in $C_{2}([0,1])$ (Lemma 3.1), we have that $F_{1}([0,1]) \subset \beta[5$, p. 78].

The following result is analogous to $[15,4.6]$ and its proof is very similar if we use Corollary 3.2.

TheOREM 3.3. $\mathrm{PHS}_{2}([0,1])$ is homeomorphic to $[0,1]^{4}$.

TheOrem 3.4. $\mathrm{PHS}_{n}\left(S^{1}\right)$ does not have the fixed point property.

Proof. Since $C\left(S^{1}\right)$ is a 2-cell [17, Example 0.55], we have that $C\left(S^{1}\right)$ is an absolute retract [20, Théorème $\left.\mathrm{II}_{m}\right]$. Then since $C\left(S^{1}\right) \subset C_{n}\left(S^{1}\right)$, there exists a retraction $G: C_{n}\left(S^{1}\right) \rightarrow C\left(S^{1}\right)$. Hence, $G\left(F_{1}\left(S^{1}\right)\right)=F_{1}\left(S^{1}\right)$. Then there exists a map $G_{*}: C_{n}\left(S^{1}\right) / F_{1}\left(S^{1}\right) \rightarrow C\left(S^{1}\right) / F_{1}\left(S^{1}\right)$ such that $G_{*} \circ q_{S^{1}}^{n}=q_{S^{1}} \circ G$, where $q_{S^{1}}^{n}: C_{n}\left(S^{1}\right) \rightarrow C_{n}\left(S^{1}\right) / F_{1}\left(S^{1}\right)$ and $q_{S^{1}}: C\left(S^{1}\right) \rightarrow$ $C\left(S^{1}\right) / F_{1}\left(S^{1}\right)$ are the quotient maps [2, Theorem 7.7, p. 17]. Observe that $P H S_{n}\left(S^{1}\right)=C_{n}\left(S^{1}\right) / F_{1}\left(S^{1}\right)$ and $H S\left(S^{1}\right)=C\left(S^{1}\right) / F_{1}\left(S^{1}\right)$. Since $G$ is a retraction, it is not difficult to see that $G_{*}: P H S_{n}\left(S^{1}\right) \rightarrow H S\left(S^{1}\right)$ is a retraction. Thus, since $H S\left(S^{1}\right)$ is a 2 -sphere [18, p. 131], $P H S_{n}\left(S^{1}\right)$ does not have the fixed point property.

REMARK 3.5. Let us observe that it is not known if $H S_{n}\left(S^{1}\right)$ has the fixed point property for $n \geq 2$.

THEOREM 3.6. If $X$ is a contractible continuum and $n$ is a positive integer, then $\mathrm{PHS}_{n}(X)$ is contractible.

Proof. Let $F: X \times[0,1] \rightarrow X$ be a map such that $F(x, 0)=x$ and $F(x, 1)=p$ for all $x \in X$ and some $p \in X$. Define $G: C_{n}(X) \times[0,1] \rightarrow C_{n}(X)$ by $G(A, t)=F(A \times\{t\})$. Then $G$ is continuous, $G(A, 0)=A, G(A, 1)=\{p\}$ for each $A \in C_{n}(X)$ and if $A \in F_{1}(X)$, then $G(A, t) \in F_{1}(X)$ for all $t \in[0,1]$. Let $R: P H S_{n}(X) \times[0,1] \rightarrow P H S_{n}(X)$ given by

$$
R(\chi, t)=\left\{\begin{array}{cl}
F_{X}^{n} & \text { if } \chi=F_{X}^{n} \\
q_{X}^{n}\left(G\left(\left(q_{X}^{n}\right)^{-1}(\chi), t\right)\right) & \text { if } \chi \neq F_{X}^{n} .
\end{array}\right.
$$

Then $R$ is continuous $[2,4.3, \mathrm{p} .126]$. Note that $R(\chi, 0)=\chi$ and $R(\chi, 1)=F_{X}^{n}$ for every $\chi \in P H S_{n}(X)$. Therefore, $P H S_{n}(X)$ is contractible. 
Corollary 3.7. $P H S_{n}([0,1])$ is not homeomorphic to $P H S_{n}\left(S^{1}\right)$, for any $n \geq 2$.

Proof. Since $[0,1]$ is contractible, by Theorem 3.6, $P H S_{n}([0,1])$ is contractible. Since $P H S_{n}\left(S^{1}\right)$ has a retract homeomorphic to $S^{2}$ (see proof of Theorem 3.4), $P H S_{n}\left(S^{1}\right)$ is not contractible. Therefore, $P H S_{n}([0,1])$ is not homeomorphic to $\mathrm{PHS}_{n}\left(S^{1}\right)$.

THEOREM 3.8. If $X$ is a continuum, then $P H S_{n}(X)$ is uniformly pathwise connected for any integer $n \geq 2$.

Proof. It is known that $C_{n}(X)$ is a continuous image of the Cantor fan [6, Remark, p. 29]. Hence, $P H S_{n}(X)$ is a continuous image of the Cantor fan. Therefore, $P H S_{n}(X)$ is uniformly pathwise connected [7, 3.5].

The proof of the following result is similar to that in [12, Theorem 3.6].

THEOREM 3.9. If $X$ is a finite-dimensional continuum and $n \geq 2$, then $\operatorname{dim}\left(C_{n}(X)\right)=\operatorname{dim}\left(P H S_{n}(X)\right)$.

Corollary 3.10. $\operatorname{dim}\left(P H S_{n}([0,1])\right)=\operatorname{dim}\left(P H S_{n}\left(S^{1}\right)\right)=2 n$ for each integer $n \geq 2$.

Proof. Since $\operatorname{dim}\left(C_{n}([0,1])\right)=\operatorname{dim}\left(C_{n}\left(S^{1}\right)\right)=2 n$, for any positive integer $n$ [13, Theorem 6.8.10], the result follows from Theorem 3.9.

The techniques used to prove the following results are similar to the ones used in $[12,3.7$ and 3.8$]$.

Let us recall that a simple $m$-od in a continuum $X$ is the union of $m$ arcs emanating from a single point, $v$, and otherwise disjoint from each other.

Theorem 3.11. Let $X$ be a continuum and let $n \geq 2$. Then:

(a) $P H S_{n}(X)$ always contains n-cells.

(b) If $X$ contains $n$ pairwise disjoint decomposable subcontinua, then $\mathrm{PHS}_{n}(X)$ contains $2 n$-cells.

(c) If $X$ contains a simple $m$-od, then PHS $S_{n}(X)$ contains $(2 n+m-2)$ cells.

The proof of the following theorem is similar to the one given for [12, 3.9].

A finite-dimensional continuum $X$ is a Cantor manifold if for any subset $A$ of $X$ such that $\operatorname{dim}(A) \leq \operatorname{dim}(X)-2$, then $X \backslash A$ is connected.

THEOREM 3.12. Let $X$ be a continuum, and let $n \geq 2$. If $C_{n}(X)$ is a finite-dimensional Cantor manifold such that $\operatorname{dim}\left(C_{n}(X)\right) \geq n+2$, then $\mathrm{PHS}_{n}(\mathrm{X})$ is a finite-dimensional Cantor manifold.

QUESTION 3.1. Is it true that, for each continuum $X$ and each $n \geq 2$, $\operatorname{dim}\left(C_{n}(X)\right) \geq n+2$ ? 
Corollary 3.13. $\mathrm{PHS}_{n}([0,1])$ and $\mathrm{PH} S_{n}\left(S^{1}\right)$ are $2 n$-dimensional Cantor manifolds, for each integer $n \geq 2$.

Proof. It is known that $C_{n}([0,1])$ and $C_{n}\left(S^{1}\right)$ are $2 n$-dimensional Cantor manifolds $[14$, Theorem 4.6]. Since $n \geq 2,2 n \geq n+2$. Hence, the result follows from Theorem 3.12.

\section{LOCAL CONNECTEDNESS}

In this section we present results about the $n$-fold pseudo-hyperspace suspensions of locally connected continua.

The techniques used to prove the following results are similar to the ones used in [12, 5.2 and 5.3].

Theorem 4.1. Let $X$ be a continum and let $n \geq 2$. Then:

(a) $X$ is locally connected if and only if $P H S_{n}(X)$ is locally connected.

(b) If $X$ is a contractible locally connected continuum without free arcs, then $\mathrm{PHS}_{n}(X)$ is homeomorphic to the Hilbert cube. In particular, $C_{n}(X), H S_{n}(X)$ and $P H S_{n}(X)$ are homeomorphic.

COROLlary 4.2. The $n$-fold pseudo-hyperspace suspensions of the Hilbert cube are homeomorphic to the Hilbert cube for each integer $n \geq 2$.

EXAMPLE 4.3. There exists a locally connected continuum $X$ without free arcs such that $P H S_{n}(X)$ is not homeomorphic to the Hilbert cube (see [3, Example 5.3 ]).

The following theorem shows that $[0,1]$ and $S^{1}$ have unique $n$-fold pseudohyperspace suspensions.

Theorem 4.4. Let $X$ be a continuum, and let $n \geq 2$. If $P H S_{n}(X)$ is homeomorphic to $P H S_{n}([0,1])$, then $X$ is homeomorphic to $[0,1]$. Also, if $\mathrm{PHS}_{n}(X)$ is homeomorphic to $\mathrm{PH} S_{n}\left(S^{1}\right)$, then $X$ is homeomorphic to $S^{1}$.

Proof. Suppose $P H S_{n}(X)$ is homeomorphic to $P H S_{n}([0,1])$. By an argument similar to the one given in $[12,5.7]$, we conclude that $X$ is homeomorphic to either $[0,1]$ or $S^{1}$. Since $\operatorname{PHS}_{n}([0,1])$ is not homeomorphic to $P H S_{n}\left(S^{1}\right)$ (Corollary 3.7), we have that $X$ is homeomorphic to $[0,1]$.

In a similar way, we can show that if $P H S_{n}(X)$ is homeomorphic to $P H S_{n}\left(S^{1}\right)$, then $X$ is homeomorphic to $S^{1}$.

A finite graph is a continuum that can be written as the union of finitely many arcs, any two of which can intersect in at most one or both of their end points.

Lemma 4.5. Let $X$ be a continuum, and let $n \geq 2$. Then $P H S_{n}(X)$ is locally connected and $\operatorname{dim}\left(P H S_{n}(X)\right)<\infty$ if and only if $X$ is a finite graph. 
Proof. The result follows from Theorem 4.1 (a), Theorem 3.9 and [11, Theorem 5.1].

Corollary 4.6. Let $X$ be a continuum, let $n \geq 2$ and let $G$ be a finite graph. If $\mathrm{PHS}_{n}(X)$ is homeomorphic to $\mathrm{PH} S_{n}(G)$, then $X$ is a finite graph.

QUESTION 4.1. Let $G_{1}$ and $G_{2}$ be a finite graphs, and let $n \geq 2$. If PHS $S_{n}\left(G_{1}\right)$ is homeomorphic to PHS $S_{n}\left(G_{2}\right)$, then is $G_{1}$ homeomorphic to $G_{2}$ ?

\section{Aposyndesis}

In this section we show that $\mathrm{PHS}_{n}(X)$ is zero-dimensional aposyndetic.

Theorem 5.1. Let $X$ be a continuum, and let $n \geq 2$. Then $P H S_{n}(X)$ has property (b).

Proof. It is known that $C_{n}(X)$ has property $(b)$ [10, 4.8]. Since the map $q_{X}^{n}$ is monotone, and a monotone image of a continuum satisfying property (b) has property $(b)$ [8, Theorem 2, p. 434], we have that $q_{X}^{n}\left(C_{n}(X)\right)=$ $P H S_{n}(X)$ has property $(b)$.

Corollary 5.2. $\mathrm{PHS}_{n}(X)$ is unicoherent.

Proof. Since $\mathrm{PHS}_{n}(X)$ has property (b) (Theorem 5.1), the result follows from [5, Lemma 19.7].

A continuum is said to be colocally connected at $p \in X$ provided for each open set $V$ of $X$ such that $p \in V$, there exist an open set $W$ of $X$ such that $p \in W \subset V$ and $X \backslash W$ is connected. The continuum $X$ is colocally connected provided is collocaly connected at each one of its points.

Theorem 5.3. Let $X$ be a continuum, and let $n \geq 2$. Then $P H S_{n}(X)$ is colocally connected.

Proof. Let $\chi \in P H S_{n}(X)$ and let $\mathcal{U}$ be an open subset of $P H S_{n}(X)$ such that $\chi \in \mathcal{U}$. We consider two cases.

CASE 1. $\chi=F_{X}$. Then $\left(q_{X}^{n}\right)^{-1}(\chi)=F_{1}(X)$ and $\left(q_{X}^{n}\right)^{-1}(\mathcal{U})$ is open in $C_{n}(X)$ such that $F_{1}(X) \subset\left(q_{X}^{n}\right)^{-1}(\mathcal{U})$. We have that, for each $x \in X$, there exists an open set $V_{X}$ in $X$ such that $\{x\} \subset\left\langle V_{X}\right\rangle \subset\left(q_{X}^{n}\right)^{-1}(\mathcal{U})$. Let $\mathcal{V}$ $=\bigcup_{x \in X} q_{X}^{n}\left(\left\langle V_{X}\right\rangle\right)$. Hence, $\mathcal{V}$ is an open set in $P H S_{n}(X)$ such that $F_{X}^{n} \in \mathcal{V} \subset \mathcal{U}$. To show that $P H S_{n}(X) \backslash \mathcal{V}$ is connected, we need to prove that any element of $P H S_{n}(X) \backslash \mathcal{V}$ can be joined to $T_{X}^{n}$ with an arc in $P H S_{n}(X) \backslash \mathcal{V}$. For this, let $\xi \in P H S_{n}(X) \backslash \mathcal{V}$. We observe that $\left(q_{X}^{n}\right)^{-1}(\xi) \in C_{n}(X) \backslash\left(\bigcup_{x \in X}\left\langle V_{X}\right\rangle\right)$. Let $\gamma$ be an order arc in $C_{n}(X)$ such that $\gamma(0)=\left(q_{X}^{n}\right)^{-1}(\xi)$ and $\gamma(1)=X$. Then, $\gamma \cap\left(\bigcup_{x \in X}\left\langle V_{X}\right\rangle\right)=\emptyset$. Hence, $q_{X}^{n}(\gamma)$ is an arc in $P H S_{n}(X)$ such that 
$\left(q_{X}^{n}(\gamma)\right)(0)=\xi$ and $\left(q_{X}^{n}(\gamma)\right)(1)=T_{X}^{n}$. We note that $q_{X}^{n}(\gamma) \cap \mathcal{V}=\emptyset$. Hence, $q_{X}^{n}(\gamma) \subset P H S_{n}(X) \backslash \mathcal{V}$. Since $\xi$ is an arbitrary element of $P H S_{n}(X) \backslash \mathcal{V}$, we have that $P H S_{n}(X) \backslash \mathcal{V}$ is connected.

CASE 2. $\chi \neq F_{X}^{n}$. We note that $\left(q_{X}^{n}\right)^{-1}(\mathcal{U})$ is open in $C_{n}(X)$ such that $\left(q_{X}^{n}\right)^{-1}(\chi) \in\left(q_{X}^{n}\right)^{-1}(\mathcal{U})$. Let $\varepsilon>0$ be such that $\mathcal{V}_{\varepsilon}^{\mathcal{H}}\left(\left(q_{X}^{n}\right)^{-1}(\chi)\right) \subset\left(q_{X}^{n}\right)^{-1}(\mathcal{U})$ and $\mathcal{V}_{\varepsilon}^{\mathcal{H}}\left(\left(q_{X}^{n}\right)^{-1}(\chi)\right) \cap F_{1}(X)=\emptyset$. Since $C_{n}(X)$ is colocally connected $[10$, Theorem 5.1], there exists an open set $W$ in $C_{n}(X)$ such that $\left(q_{X}^{n}\right)^{-1}(\chi) \in$ $W \subset \mathcal{V}_{\varepsilon}^{\mathcal{H}}\left(\left(q_{X}^{n}\right)^{-1}(\chi)\right)$ and $C_{n}(X) \backslash W$ is connected. Hence, $q_{X}^{n}(W)$ is open in $P H S_{n}(X)$ such that $\chi \in q_{X}^{n}(W) \subset \mathcal{U}$ and, moreover, $P H S_{n}(X) \backslash q_{X}^{n}(W)=$ $q_{X}^{n}\left(C_{n}(X) \backslash W\right)$. By the continuity of $q_{X}^{n}$, we have that $P H S_{n}(X) \backslash q_{X}^{n}(W)$ is connected. Therefore, $P H S_{n}(X)$ is colocally connected.

Clearly, colocal connectedness implies aposyndesis. Hence, we have the following result as a consequence of Theorem 5.3.

Corollary 5.4. If $X$ is a continuum and $n \geq 2$, then $P H S_{n}(X)$ is aposyndetic.

A continuum $X$ is said to be zero-dimensional aposyndetic provided that for each zero-dimensional closed subset $A$ of $X$ and for each $p \in X \backslash A$, there exists a subcontinuum $M$ of $X$ such that $p \in \operatorname{Int}_{X}(M)$ and $M \cap A=\emptyset$.

Theorem 5.5. Let $X$ be a continuum, and let $n \geq 2$. Then $P H S_{n}(X)$ is zero-dimensional aposyndetic.

Proof. Let $\mathcal{Z}$ be a nonempty zero-dimensional closed subset of $P H S_{n}(X)$ and let $\chi \in P H S_{n}(X) \backslash \mathcal{Z}$. We consider three cases.

CASE 1. $\chi \in \operatorname{PHS}_{n}(X) \backslash\left\{F_{X}^{n}, T_{X}^{n}\right\}$. Note that $\left(q_{X}^{n}\right)^{-1}(\mathcal{Z})$ is closed in $C_{n}(X)$ and $\left(q_{X}^{n}\right)^{-1}(\chi) \notin\left(q_{X}^{n}\right)^{-1}(\mathcal{Z})$. Let $\varepsilon>0$ be such that

$$
C l_{C_{n}(X)}\left(\mathcal{V}_{\varepsilon}^{\mathcal{H}}\left(\left(q_{X}^{n}\right)^{-1}(\chi)\right)\right) \cap F_{1}(X)=\emptyset
$$

and

$$
C l_{P H S_{n}(X)}\left(q_{X}^{n}\left(\mathcal{V}_{\varepsilon}^{\mathcal{H}}\left(\left(q_{X}^{n}\right)^{-1}(\chi)\right)\right) \cap \mathcal{Z}=\emptyset .\right.
$$

Now, let $\mathcal{Z}^{\prime}=\mathcal{Z} \backslash\left\{F_{X}^{n}\right\}$. Hence, $\operatorname{dim}\left(\mathcal{Z}^{\prime}\right) \leq 0$ and

$$
\mathcal{V}_{\varepsilon}^{\mathcal{H}}\left(\left(q_{X}^{n}\right)^{-1}(\chi)\right) \cap\left(q_{X}^{n}\right)^{-1}\left(\mathcal{Z}^{\prime}\right)=\emptyset .
$$

Then there exists a subcontinuum $W$ of $C_{n}(X)$ such that $\left(q_{X}^{n}\right)^{-1}(\chi) \in$ $\operatorname{Int}_{C_{n}(X)}(W) \subset W \subset C_{n}(X) \backslash\left(q_{X}^{n}\right)^{-1}\left(\mathcal{Z}^{\prime}\right)$ [16, Theorem 2.6]. Note that in the proof of [16, Theorem 2.6], the fact that the zero-dimensional set $\mathcal{Z}$ is closed is used only to construct an open set about a point whose closure $\operatorname{missed} \mathcal{Z}$. To construct $W$, only the fact that $\operatorname{dim}\left(\mathcal{Z}^{\prime}\right) \leq 0$ is used (see [16, Lemma 2.5]). Observe that $W$ can be constructed in such a way that 
$W \cap F_{1}(X)=\emptyset$ (in the proof of [16, Theorem 2.6], the continuum constructed is contained in a set of the form $\left\{A \in C_{n}(X): A \in \mu^{-1}([s, t])\right\}$, where $\mu$ is a Whitney map and $0 \leq s<t \leq 1$. Since $\left(q_{X}^{n}\right)^{-1}(\chi) \notin F_{1}(X)$, we can take $s \neq 0)$. Hence, $q_{X}^{n}(W)$ is a subcontinuum of $P H S_{n}(X)$ such that $\chi \in \operatorname{Int}_{P H S_{n}(X)}\left(q_{X}^{n}(W)\right) \subset q_{X}^{n}(W) \subset P H S_{n}(X) \backslash \mathcal{Z}^{\prime}$. Since $W \cap F_{1}(X)=\emptyset$, we have that $F_{X}^{n} \notin q_{X}^{n}(W)$. Therefore, $q_{X}^{n}(W) \subset P H S_{n}(X) \backslash \mathcal{Z}$.

CASE 2. $\chi=T_{X}^{n}$. Since $P H S_{n}(X)$ is normal, there exists an open subset $\mathcal{U}$ in $P H S_{n}(X)$ such that $C l_{P H S_{n}(X)}(\mathcal{U}) \cap \mathcal{Z}=\emptyset$ and $T_{X}^{n} \in \mathcal{U}$. From the existence of order $\operatorname{arcs}$ in $C_{n}(X)$, it is easy to see that $C_{n}(X)$ is locally connected at $X$. Thus, $P H S_{n}(X)$ is locally connected at $T_{X}^{n}$. Hence, there exists an open and connected subset $\mathcal{V}$ of $P H S_{n}(X)$ such that $T_{X}^{n} \in \mathcal{V} \subset \mathcal{U}$. We obtain that $C l_{P H S_{n}(X)}(\mathcal{V}) \cap \mathcal{Z}=\emptyset$. Therefore, $C l_{P H S_{n}(X)}(\mathcal{V})$ is a subcontinuum of $P H S_{n}(X)$ such that $T_{X}^{n} \in \operatorname{Int}\left(C l_{P H S_{n}(X)}(\mathcal{V})\right) \subset C l_{P H S_{n}(X)}(\mathcal{V}) \subset$ $P H S_{n}(X) \backslash \mathcal{Z}$.

CASE 3. $\chi=F_{X}^{n}$. Let $\mathcal{Z}^{\prime}=\left(q_{X}^{n}\right)^{-1}(\mathcal{Z})$. Hence, $\mathcal{Z}^{\prime}$ is a closed subset of $C_{n}(X)$ such that $\operatorname{dim}\left(\mathcal{Z}^{\prime}\right)=0$ and $\mathcal{Z}^{\prime} \cap F_{1}(X)=\emptyset$. Thus, for each $x \in X$, there exists a subcontinuum $W_{x}$ of $C_{n}(X)$ such that $\{x\} \in$ $\operatorname{Int}_{C_{n}(X)}\left(W_{x}\right) \subset W_{x} \subset C_{n}(X) \backslash \mathcal{Z}^{\prime}\left[16\right.$, Theorem 2.6]. Since $F_{1}(X)$ is compact, there exist a finite cover $\left\{W_{x}^{i}\right\}_{i=1}^{k}$ such that $F_{1}(X) \subset \operatorname{Int}_{C_{n}(X)}\left(W_{x}^{i}\right)$. Let $W=\bigcup\left\{W_{x}^{i}: x \in X\right\}$. We have that, $W$ is a subcontinuum of $C_{n}(X)$ such that $F_{1}(X) \subset \operatorname{Int}_{C_{n}(X)}(W) \subset W \subset C_{n}(X) \backslash \mathcal{Z}^{\prime}$. Therefore, $q_{X}^{n}(W)$ is a subcontinuum of $P H S_{n}(X)$ such that $F_{X}^{n} \in \operatorname{Int}_{P H S_{n}(X)}\left(q_{X}^{n}(W)\right) \subset q_{X}^{n}(W) \subset$ $P H S_{n}(X) \backslash \mathcal{Z}$.

\section{Points that arCWise DisconneCt}

In this section we show that a continuum $X$ is indecomposable if and only if by removing the set $\left\{T_{X}^{n}, F_{X}^{n}\right\}$ from $P H S_{n}(X)$ it becomes arcwise disconnected.

Given a continuum $X$ and any point $p \in X$, the composant of $p$ in $X$ is the set

$\{x \in X$ : there is a proper subcontinuum $A$ of $X$ such that $p, x \in A\}$.

By a composant of $X$ we mean a composant of some point in $X[19,5.20]$.

Lemma 6.1. Let $n \geq 2$ be an integer. If $X$ is a decomposable continuum, then $C_{n}(X) \backslash\left(\{X\} \cup F_{1}(X)\right)$ is arcwise connected.

Proof. Since $C_{n}(X) \backslash\left(\{X\} \cup F_{n}(X)\right)$ is arcwise connected [12, Lemma 6.1], note that it suffices to show that any point $x$ in $F_{n}(X) \backslash F_{1}(X)$ can be joined to some point $A$ of $C_{n}(X) \backslash\left(\{X\} \cup F_{n}(X)\right)$ with an arc in $C_{n}(X) \backslash\left(\{X\} \cup F_{1}(X)\right)$.

Let $\boldsymbol{x}=\left\{x_{1}, \ldots, x_{k}\right\}$ in $F_{n}(X) \backslash F_{1}(X)$. Since $X$ is normal, there exist $U_{1}, \ldots, U_{k}$, open sets in $X$ such that $x_{i} \in U_{i}$, for each $i \in\{1, \ldots, k\}$ and 
$U_{i} \cap U_{j}=\emptyset, i \neq j$, where $i, j \in\{1, \ldots, k\}$. Then there exist subcontinua $B_{1}, \ldots, B_{k}$ of $X$ such that $x_{i} \in B_{i} \subset U_{i}$, for each $i \in\{1, \ldots, k\}$ [19, Corollary 5.5]. Note that $\bigcup_{i=1}^{n} B_{i} \in C_{n}(X) \backslash\left(\{X\} \cup F_{n}(X)\right) \subset C_{n}(X) \backslash\left(\{X\} \cup F_{1}(X)\right)$.

Let $A=\bigcup_{i=1}^{n} B_{i}$. Since $\boldsymbol{x} \subset A$, by [17, Theorem 1.8], there exists an order $\operatorname{arc} \alpha:[0,1] \rightarrow C_{n}(X) \backslash\left(\{X\} \cup F_{1}(X)\right)$ such that $\alpha(0)=\boldsymbol{x}$, and $\alpha(1)=A$. Therefore, $C_{n}(X) \backslash\left(\{X\} \cup F_{1}(X)\right)$ is arcwise connected.

Theorem 6.2. Let $n$ be a positive integer. A continuum $X$ is indecomposable if and only if $P H S_{n}(X) \backslash\left\{T_{X}^{n}, F_{X}^{n}\right\}$ is not arcwise connected.

Proof. Suppose $X$ is an indecomposable continuum. Then, $C_{n}(X) \backslash\{X\}$ is not arcwise connected [10, Theorem 6.3]. Hence, $C_{n}(X) \backslash\left(\{X\} \cup F_{1}(X)\right)$ is not arcwise connected either (any two nondegenerate subcontinua $A$ and $B$ of $X$, contained in two different composants of $X$, are in two different arc components of $\left.C_{n}(X) \backslash\left(\{X\} \cup F_{1}(X)\right)\right)$. Since $C_{n}(X) \backslash\left(\{X\} \cup F_{1}(X)\right)$ is homeomorphic to $P H S_{n}(X) \backslash\left\{T_{X}^{n}, F_{X}^{n}\right\}$ (Remark 2.3), $P H S_{n}(X) \backslash\left\{T_{X}^{n}, F_{X}^{n}\right\}$ is not arcwise connected.

Suppose $X$ is a decomposable continuum. By Lemma 6.1, the space $C_{n}(X) \backslash\left(\{X\} \cup F_{1}(X)\right)$ is arcwise connected. Since $C_{n}(X) \backslash\left(\{X\} \cup F_{1}(X)\right)$ is homeomorphic to $P H S_{n}(X) \backslash\left\{T_{X}^{n}, F_{X}^{n}\right\}$ (Remark 2.3), $P H S_{n}(X) \backslash\left\{T_{X}^{n}, F_{X}^{n}\right\}$ is arcwise connected.

QUESTION 6.1. If $X$ is an indecomposable continuum and $n \geq 2$, then is $\mathrm{PHS}_{n}(\mathrm{X})$ homeomorphic to $H S_{n}(X)$ ?

\section{ACKNOWLEDGEMENTS.}

The author thanks the referee for his/her valuable suggestions which improved the paper.

\section{REFERENCES}

[1] D. Curtis and N. T. Nhu, Hyperspaces of finite subsets which are homeomorphic to $\aleph_{0}$-dimensional linear metric spaces, Topology Appl. 19 (1985), 251-260.

[2] J. Dugundji, Topology, Allyn and Bacon, Inc., Boston, Mass, 1966.

[3] R. Escobedo, M. de Jesús López and S. Macías, On the hyperspace suspension of a continuum, Topology Appl. 138 (2004), 109-124.

[4] A. Illanes, The hyperspace $\mathrm{C}_{2}(\mathrm{X})$ for a finite graph $\mathrm{X}$ is unique, Glas. Mat. Ser. III 37(57) (2002), 347-363.

[5] A. Illanes and S. B. Nadler Jr., Hyperspaces. Fundamentals and Recent Advances, Monographs and Textbooks in Pure and Applied Mathematics 216, Marcel Dekker, Inc., New York, 1999.

[6] J. L. Kelley, Hyperspaces of a continuum, Trans. Amer. Math. Soc. 52 (1942), 22-36.

[7] W. Kuperberg, Uniformly pathwise connected continua, in: N.M. Stavrakas, K.R. Allen (Eds.), Studies in Topology, Proc. Conf. Univ. North Carolin, Charlotte, NC, 1974, Academic Press, New York, 1975, 315-324.

[8] K. Kuratowski, Topology. Vol. II, Academic Press, New York-London, 1968. 
[9] S. Macías, Aposyndetic properties of symmetric products of continua, Topology Proc. 22 (1997), 281-296.

[10] S. Macías, On the hyperspaces $\mathrm{C}_{n}(\mathrm{X})$ of a continuum X, Topology Appl. 109 (2001), 237-256.

[11] S. Macías, On the hyperspaces $\mathrm{C}_{n}(\mathrm{X})$ of a continuum X, II Topology Proc. 25 (2000), 255-276.

[12] S. Macías, On the n-fold hyperspace suspension of continua, Topology Appl. 138 (2004), 125-138.

[13] S. Macías, Topics on Continua, Pure and Applied Mathematics Series, vol. 275, Chapman and Hall/CRC, Taylor and Francis Group, Boca Raton, London, New York, Singapore, 2005.

[14] S. Macías and S. B. Nadler Jr., n-fold hyperspaces, cones and products, Topology Proc. 26 (2001/2002), 255-270.

[15] S. Macías and S. B. Nadler Jr., Absolute n-fold hyperspace suspensions, Colloquium Mathematicum 105 (2006), 221-231.

[16] J. M. Martínez-Montejano, Zero-dimensional closed set aposyndesis and hyperspaces, Houston J. Math. 32 (2006), 1101-1105.

[17] S. B. Nadler Jr., Hyperspaces of Sets, Monographs Textbooks Pure Appl. Math. 49, Marcel Dekker, Inc., New York-Basel, 1978.

[18] S. B. Nadler Jr., A fixed point theorem for hyperspace suspensions, Houston J. Math. 5 (1979), 125-132.

[19] S. B. Nadler Jr., Continuum theory. An introduction, Monographs Textbooks Pure Applied Mathematics 158, Marcel Dekker, Inc., New York, 1992.

[20] M. Wojdislawski, Rétractes absolus et hyperespaces des continus, Fund. Math. 32 (1939), 184-192

J. C. Macías

Facultad de Ciencias Físico Matemáticas, BUAP

Ave. San Claudio y Río Verde, Ciudad Universitaria, San Manuel

Puebla Pue. C.P. 72570

México

E-mail: jcmacias@fcfm.buap.mx

Received: 25.5.2007.

Revised: 24.10 .2007 . \& 24.12.2007. 